\title{
Condiciones hidrográficas y climatológicas en el Caribe Sur de Costa Rica durante El Niño 2014-2016
}

\section{Hydrographic and climatological conditions in the southern Caribbean of Costa Rica during El Niño 2014-2016}

\author{
Carlos Luis Brenes-Rodriguez ${ }^{1}$, Rosario Benavides-Morera ${ }^{1}$, Juan Pablo Salazar-Ceciliano y \\ Luis Fernando Alvarado-Gamboa ${ }^{2}$
}

\begin{abstract}
RESUMEN
En mayo, junio, septiembre y octubre del año 2014 y junio, agosto, septiembre y noviembre del año 2015, se realizaron campañas hidrográficas en el Caribe Sur de Costa Rica, con el objetivo de determinar la influencia del fenómeno de El Niño sobre esta región. Con un perfilador se determinó en 23 estaciones la temperatura, la salinidad y la clorofila $a$. La temperatura superficial se ubicó entre 26.6 y $30^{\circ} \mathrm{C}$, las salinidades superficiales entre 29 y 33.5 UPS y la clorofila $a$ superficial varió entre $1.1 \mathrm{mg} \mathrm{m}^{-3}$ y $0.1 \mathrm{mg} \mathrm{m}^{-3}$. Existe una capa de mezcla delgada $\left(\mathrm{T} \sim 29^{\circ} \mathrm{C}\right)$, la cual no sobrepasó los 20 $\mathrm{m}$ de profundidad en el 2014 y se extendió hasta los $40 \mathrm{~m}$ en septiembre y noviembre del año 2015. Los índices termohalinos del Agua Superficial del Caribe presentaron una disminución considerable en su salinidad por dilución debido a El Niño y un ligero aumento en su temperatura. El análisis entre una serie temporal de la temperatura superficial del mar en un punto situado $15 \mathrm{~km}$ frente a Cahuita y el Índice Oceánico de El Niño (ONI) determinaron que la zona estudiada presenta una señal de calentamiento con un desfase de 7 meses posterior a la presencia de El Niño en el Océano Pacífico Tropical. Durante El Niño 2014-2016 el área de estudio presentó en algunos meses un exceso en las precipitaciones que superó el valor de la norma climatológica en más de un 50\%.
\end{abstract}

Palabras claves: Gandoca, Cahuita, Caribe, Costa Rica, El Niño.

\begin{abstract}
In May, June, September and October 2014 and June, August, September and November 2015, hydrographic campaigns were carried out in the Southern Caribbean of Costa Rica in order to determine the influence of the El Niño phenomenon on this region. With a conductivity, temperature and depth profiler, temperature, salinity and chlorophyll $a$ were determined in 23 stations. The surface temperature was between $26.6-30^{\circ} \mathrm{C}$, surface salinities between 29-33.5 UPS, and surface chlorophyll $a$ varied between $1.1 \mathrm{mg} \mathrm{m}^{-3}-0.1 \mathrm{mg} \mathrm{m}^{-3}$. There is a thin mixture layer (T $29^{\circ} \mathrm{C}$ ), which did not exceed $20 \mathrm{~m}$ depth in 2014 and extended to $40 \mathrm{~m}$ in September and November 2015. The thermohaline indexes of the Caribbean Surface Water presented a significant decrease in its salinity by dilution due to El Niño and a slight increase in its temperature. The analysis between a temporal series of sea surface temperature at a point $15 \mathrm{~km}$ off Cahuita and the Oceanic Niño Index (ONI), determined that the area under study has a warm-up signal with
\end{abstract}

\footnotetext{
Laboratorio de Oceanografía y Manejo Costero, Universidad Nacional, Heredia, Costa Rica, Apdo. 86-3000.

Instituto Meteorológico Nacional, San José, Costa Rica. cbrenes.una@gmail.com*, rbmorera@gmail.com, jps_139@yahoo.com, lalvarado@imn.ac.cr
}

Recibido: 24 de julio de 2017

Corregido: 30 de noviembre de 2017

Aceptado: 5 de diciembre de 2017

DOI: http://dx.doi.org/10.15359/revmar.9-2.5 
a lag of 7 months following the presence of El Niño in the Tropical Pacific Ocean. During the 2014-2016 El Niño the studied area presented in some months precipitation that exceeded the climatological value by more than $50 \%$.

Keywords: Gandoca, Cahuita, Caribbean, Costa Rica, El Niño.

\section{INTRODUCCIÓN}

Las zonas costeras son reconocidas como regiones de transición entre los componentes continentales y los marinos, caracterizadas por presentar una vasta diversidad de ambientes y recursos. Estas áreas están sometidas a cambios que varían de manera amplia en la escala temporal y geográfica. Son sistemas de una alta productividad biológica que se presentan al mismo tiempo extremadamente vulnerables ante las presiones no solo antropogénicas, sino también de origen natural. La concentración poblacional con todas sus actividades que se observan a lo largo de estas zonas tiene su explicación en la abundancia y diversidad de los recursos que las caracterizan.

El tema de la variabilidad y cambio climático tiene un papel protagónico en los estudios que se realizan en las zonas costeras. La alteración en el aporte de agua dulce por escorrentía trae consigo la posibilidad de alterar los niveles de oxígeno por degradación de materia orgánica (exceso de sedimentos), la actividad de productividad primaria, así como los campos de salinidad. Adicionalmente, aumentos en la temperatura del agua de mar pueden alterar la biogeografía y poner en riesgo la salud de los sistemas de arrecifes de coral.

La región costera del Caribe de Costa Rica se caracteriza por la presencia de diversos ecosistemas como lagunas costeras, bosques de manglar, playas, lechos de pastos marinos, fondos submareales duros y blandos y arrecifes de coral, sin embargo, estos últimos son los más importantes en términos de área, riqueza de especies y valor económico (Cortés, 2016).

En el extremo sur de la costa Caribe de Costa Rica entre el Parque Nacional Cahuita y el Refugio Nacional de Vida Silvestre Gandoca-Manzanillo con la frontera de Panamá, se localizan las principales áreas de arrecifes de coral. Cortés (2016) hizo un recuento exhaustivo y una descripción amplia de los trabajos científicos que se han realizado en esta región desde 1970 hasta nuestros días. En dicho trabajo se hace referencia a los impactos que ocasionó sobre los arrecifes de coral el fenómeno de El Niño 1982-1983, uno de los más fuertes del siglo pasado. El aumento de temperatura de las aguas derivó en un blanqueamiento masivo y posterior muerte de muchas comunidades coralinas (Cortés et al. 1984). Otro evento de mortalidad de corales, producto de un calentamiento de las aguas del Caribe observado en 1995, fue reportado por Jiménez (2001). A partir del segundo semestre de 1994 y hasta abril de 1995 ocurrió un evento cálido de corta duración. A pesar de la documentación recurrente de los daños ocasionados por estos 
eventos climáticos, no existe hasta la fecha un trabajo de campo que cuantifique y describa las condiciones oceánicas y climáticas durante la fase cálida del ENOS (El Niño-Oscilación del Sur) o evento El Niño, en esta área de tanto valor ecológico.

El Niño 2014-2016 hace su aparición a partir del último trimestre del 2014, y las anomalías observadas en las diferentes variables oceánicas y atmosféricas permitieron catalogarlo como un evento fuerte, de una intensidad similar a los Niños 19821983 y 1997-1998. Su fase madura fue alcanzada a finales del 2014 e inicios del 2015, y se extendió hasta junio de este último año (NOAA, 2016). La vertiente del Caribe, con escenarios climáticos asociados a El Niño, se caracteriza por presentar cantidades de precipitación mayores de lo normal (anomalía positiva). El Instituto Meteorológico Nacional de Costa Rica (IMN) reportó para el 2015 niveles de precipitación por encima de los valores normales en el Caribe, debido a los temporales ocurridos al principio (enero-febrero) y mitad de dicho año (mayo-julio) (IMN, 2016).

Los trabajos sobre la Oceanografía Física en esta región costera del Caribe son muy escasos y no han recibido la atención necesaria por parte de los investigadores, aunque esta disciplina científica como lo plantea Cortés (2016), se espera contribuya a obtener una mejor comprensión de algunos procesos de naturaleza física que ocurren a lo largo de la costa y que impactan los ecosistemas que ahí coexisten.
El presente trabajo tuvo como objetivo primordial contribuir al conocimiento de algunos procesos oceanográficos y climáticos de esta región costera, con el propósito de que en un futuro cercano se pueda comprender de manera más integral el comportamiento de algunos recursos vivos desde un abordaje ecosistémico, en el cual el ambiente y sus variaciones juegan un papel modulador, en este caso con la presencia de la fase cálida del ENOS.

\section{METODOLOGÍA}

La región estudiada se ubicó entre el Parque Nacional Cahuita y el Refugio Nacional de Vida Silvestre GandocaManzanillo en la costa sur del Caribe de Costa Rica (Fig. 1), dos regiones de un gran interés en las agendas de conservación ambiental de este país. A lo largo de seis transectos perpendiculares a la costa se muestreó en 23 estaciones. Se realizaron ocho campañas hidrográficas: mayo, junio, septiembre y octubre del año 2014, y junio, agosto, septiembre y noviembre del año 2015.

Se utilizó un perfilador vertical CTD Sea Birds, modelo SB-19plus, con sensores adicionales a los de temperatura, conductividad y presión para medir fluorescencia y contenido de oxígeno. Se elaboraron gráficos con el programa SURFER 14 (2017). Para analizar las distribuciones verticales de temperatura, salinidad y clorofila $a$, se escogieron las estaciones $2,7,10,15$, 18 y 22 , ubicadas en la parte central de cada uno de los seis transectos perpendiculares a la costa, con lo cual se construyó un transecto paralelo a la línea de costa (Fig. 1), con la finalidad 
de observar las variaciones latitudinales de los parámetros en la columna de agua. Los transectos perpendiculares a la costa no se presentan porque las variaciones de los parámetros a lo largo de ellos no son muy evidentes por la pequeña longitud de estos.

Los datos de precipitación del

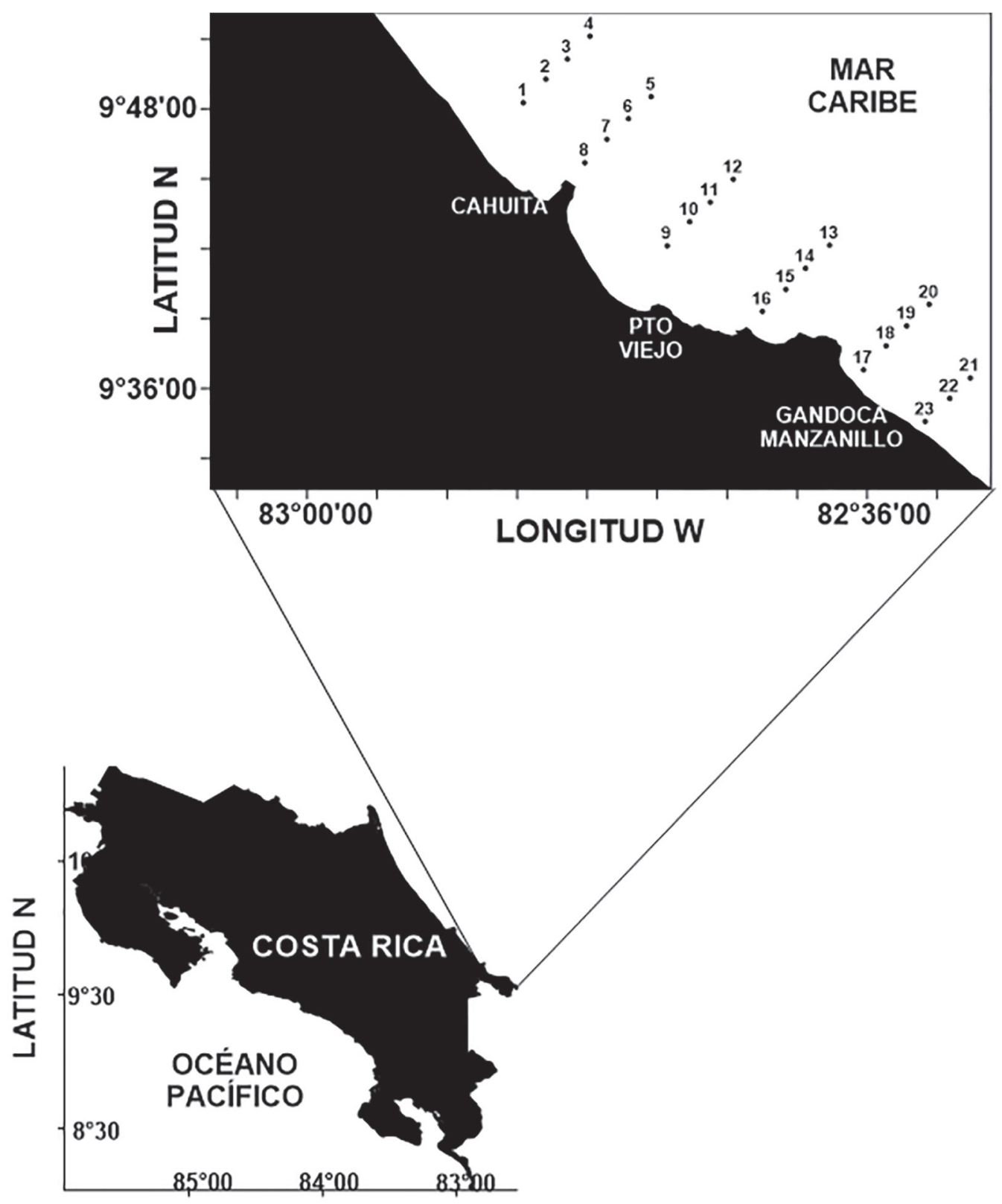

\section{LONGITUD W}

Fig. 1. Área de estudio y posición de estaciones hidrográficas

Fig. 1. Study area and position of hydrographic stations 
2014-2015 de la estación de Puerto Vargas se obtuvieron de un pluviómetro convencional (mecánico), cuyas lecturas (resolución temporal) son diarias. Esta estación está localizada en Cahuita (Talamanca, Limón), en las coordenadas $9^{\circ} 43^{\prime} \mathrm{N}, 82^{\circ} 48^{\prime} \mathrm{W}$ y a 3 m.s.n.m. La norma climatológica fue calculada con el promedio del período 1981-2010. Los datos de Sixaola provienen de un pluviómetro electrónico Campbell, cuyas lecturas son horarias. La estación se ubica en Sixaola (Talamanca, Limón), en las coordenadas $9^{\circ} 31^{\prime} \mathrm{N}, 82^{\circ} 38^{\prime} \mathrm{W}$ y a 10 m.s.n.m. La norma climatológica provisional de este lugar se calculó usando un pluviómetro convencional con el promedio del período 1979-2004. En ambos casos el pluviómetro cumple con los estándares de ubicación y exposición de la Organización Meteorológica Mundial (OMM).

Las imágenes de la temperatura superficialdelmar(TSM)corresponden a los días en los cuales se realizaron los muestreos. Se elaboraron a partir del Multi-scale Ultra-high Resolution (MUR), generado por el Jet Propulsion Laboratory (JPL-NASA), el cual se adapta a las normas establecidas por el grupo de alta resolución de temperatura superficial del mar (GHRSST), y posee una resolución espacial de un kilómetro por un kilómetro (ftp:// podaac-ftp.jpl.nasa.gov/).

Cada imagen combina datos de distintos radiómetros con observaciones in situ de la TSM mediante un algoritmo de multiescala elaborado por Chin et al. (2013), acompañado con el método de interpolación óptima mediante funciones de ondeletas (wavelets). El resultado es un producto de alta resolución espacial sin cobertura nubosa ideal para el estudio de fenómenos de pequeña escala en las zonas costeras. Los mapas de las anomalías de la temperatura superficial del mar se obtuvieron de los servicios en línea que ofrece la Oficina de Operaciones de Satélites y Productos de la National Oceanographic and Atmospheric Agency (NOAA, 2017).

Para determinar la influencia del fenómeno de El Niño sobre la TSM, se analizó una serie de tiempo de dicha variable en un punto geográfico ubicado a $15 \mathrm{~km}$ frente a Cahuita, cuyas coordenadas son $9^{\circ} 49^{\prime} 48^{\prime \prime} \mathrm{N}$ y $82^{\circ} 43^{\prime}$ 12 " W. El período analizado abarcó de junio del año 2002 a febrero del año 2017, y los datos fueron descargados del Physical Oceanography Distributed Active Archive Center (podaac) en el sitio: ftp://podaac-ftp.jpl.nasa.gov/. Se realizaron correlaciones cruzadas con el índice mensual de temperatura superficial del mar Niño 3.4, elaborado por NOAA's Earth System Research Laboratory (ESRL). Para realizar dicha comparación, a la serie de tiempo de la TSM se le calculó la señal estacional y se le restó al promedio mensual, obteniendo finalmente el residuo. A dicho residuo se le aplicó un filtro pasa-banda con una frecuencia de banda de $4.53 \times 10^{-9} \mathrm{~Hz}$ a $15.85 \times 10^{-9} \mathrm{~Hz}$ (una ventana de 2 a 7 años), de modo que la serie representa una señal con una variabilidad interanual dentro del rango de periodicidad del fenómeno de El Niño.

Asimismo, se realizó un diagrama 
dispersivo T-S, con el fin de identificar las masas de agua presentes en la columna de agua, hasta una profundidad de $110 \mathrm{~m}$.

\section{RESULTADOS}

El ámbito de temperaturas se ubicó entre $26.4^{\circ} \mathrm{C}$ y $30.5^{\circ} \mathrm{C}$ (Fig. 2). Octubre del año 2014, septiembre y noviembre del año 2015 presentaron el mayor calentamiento superficial, en correspondencia con el ciclo anual de este parámetro físico (Bernal et al. 2006; Brenes-Rodríguez \& Benavides-Morera, 2015). La Figura 3 presenta la temperatura superficial obtenida para los días de muestreo a partir del multisensor de ultra-alta resolución (MUR), generado por el Jet Propulsion Laboratory (JPL). Las imágenes corresponden a los días en que se realizaron los muestreos, y de modo general se observan patrones muy similares en relación con los resultados obtenidos in situ.

En octubre del año 2014 y más acentuadamente en septiembre del año 2015, las aguas superficiales presentaron los valores más bajos de salinidad $(29.0<\mathrm{S}<33.5)$. Asimismo, las bajas salinidades que caracterizan el extremo sur de la región estudiada reflejan la influencia de la descarga del río Sixaola (Fig. 4).

La Figura 5 presenta la precipitación mensual en las estaciones de Puerto Vargas (A) y Sixaola (B) correspondientes a los años 2014-2015, así como su valor climatológico. A partir de junio del año 2014 y hasta septiembre del año 2015, las precipitaciones estuvieron por encima de lo normal, exceptuando algunos meses. Las variaciones de las distribuciones superficiales de clorofila $a$ fluctuaron entre $0.1 \mathrm{mg} \mathrm{m}^{-3}$ y $1.2 \mathrm{mg} \mathrm{m}^{-3}$ (Fig. 6) y están dentro del ámbito reportado previamente por Brenes-Rodríguez \& Benavides-Morera (2015).

Se utilizó un transecto paralelo a la línea de costa para el análisis vertical de las variables estudiadas (estaciones $2,7,10,15,18,22)$. La mayor estratificación térmica se observó en mayo (2014) y junio (2015), y las aguas más cálidas y térmicamente homogéneas se localizaron en junio y septiembre del año 2014 (Fig. 7). Los primeros $20 \mathrm{~m}$ de la columna de agua estuvieron ocupados por aguas con temperaturas superiores a $\operatorname{los} 20^{\circ} \mathrm{C}$. La forma casi horizontal de las isotermas (excepto en agosto del 2015) evidencia la homogeneidad por niveles de los campos térmicos verticales a lo largo de todo el transecto. Con excepción de agosto del 2014 y octubre del 2014 no se observaron cambios drásticos de norte a sur en la temperatura.

Los campos verticales de salinidad en toda la columna de agua mostraron gradientes verticales significativos, exceptuando mayo del 2014 y junio del 2015, siendo más fuertes en las capas más superficiales en junio, octubre del 2014 y en agosto, septiembre del 2015 (Fig. 8). En estos meses la precipitación en la estación de Puerto Vargas superó entre 30 y $50 \%$ el valor climatológico 

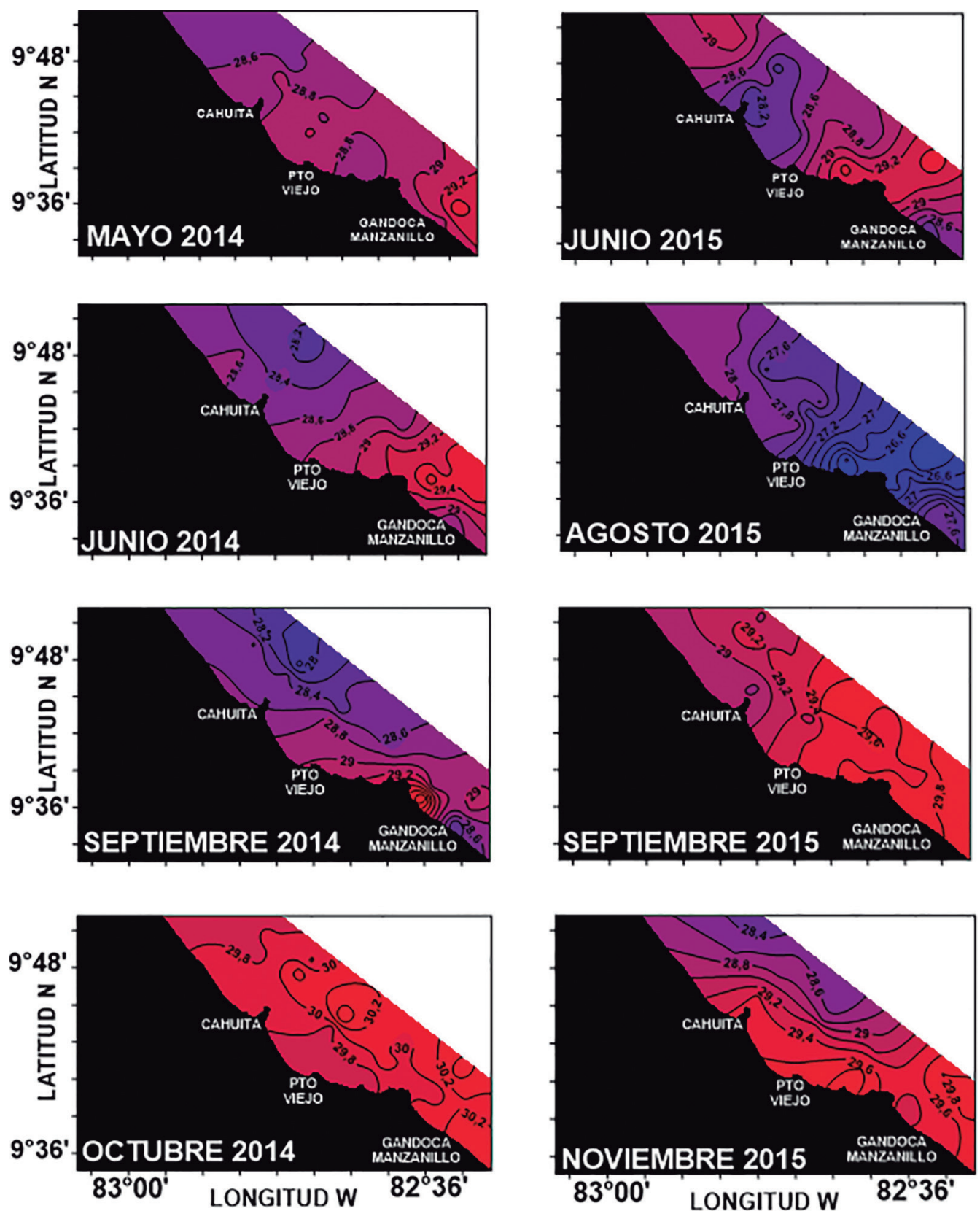

$30,029,629,228,828,628,027,627,2 \quad 26,8 \quad 26,426,0$

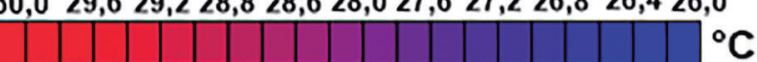

Fig. 2. Distribuciones superficiales de la temperatura del mar $\left({ }^{\circ} \mathrm{C}\right)$ para los meses de muestreo Fig. 2. Surface temperature distributions $\left({ }^{\circ} \mathrm{C}\right)$ for the sampling months 

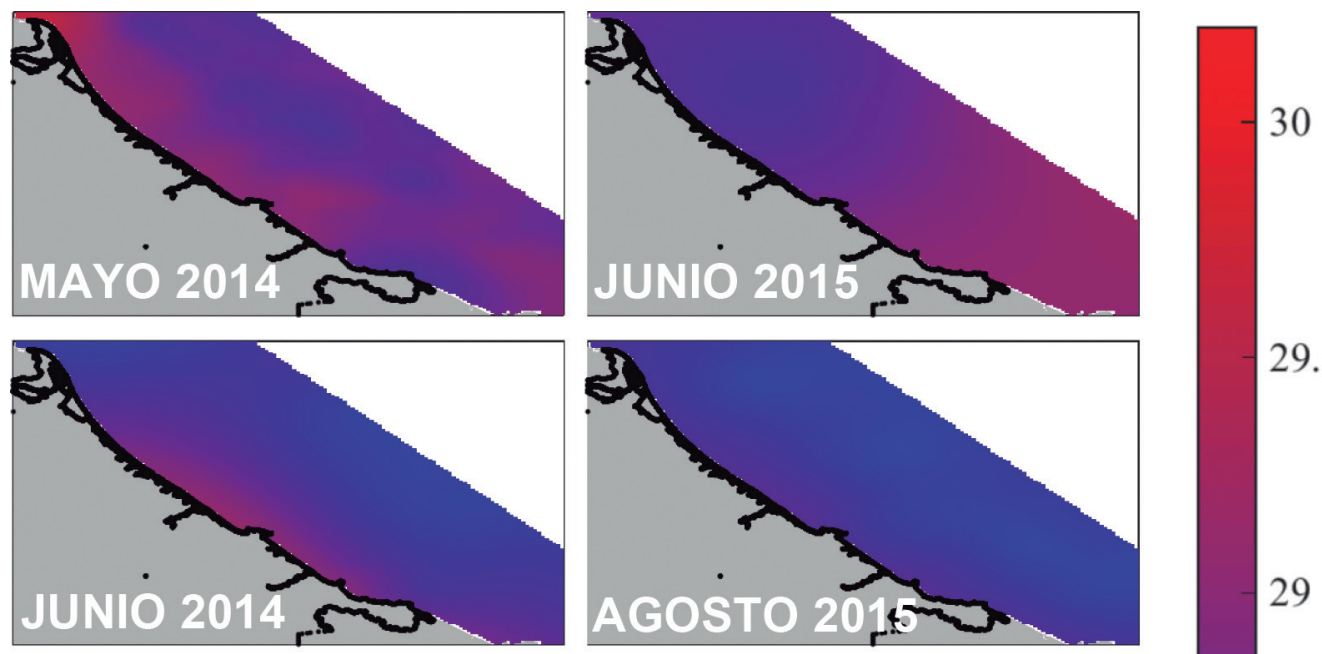

29.5
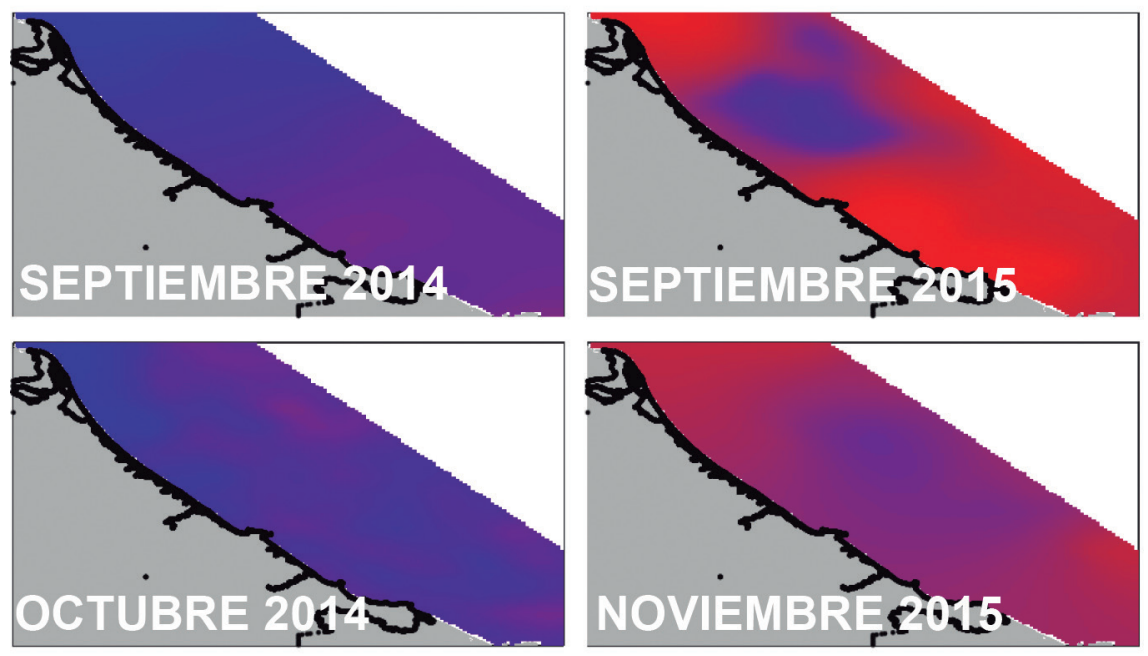

Fig. 3. Distribuciones superficiales de la temperatura del mar $\left({ }^{\circ} \mathrm{C}\right)$ para los meses de muestreo, obtenidas a partir del multisensor de ultra-alta resolución (MUR) generado por el Jet Propulsion Laboratory (JPL-NASA)

Fig. 3. Surface temperature distributions $\left({ }^{\circ} \mathrm{C}\right)$ for the sampling months, obtained from the ultra-high resolution multisensor (MUR) generated by the Jet Propulsion Laboratory (JPL-NASA)

de lluvia (Fig. 4). En el extremo sur del transecto cerca de la estación 22, se puede observar adicionalmente la influencia de la descarga del río Sixaola a través de la presencia de aguas con salinidades más bajas que el resto de la región.

El máximo de clorofila $a$ se ubicó entre los 20 y $40 \mathrm{~m}$ de profundidad (Fig. 9), con un valor cercano a 0.8 

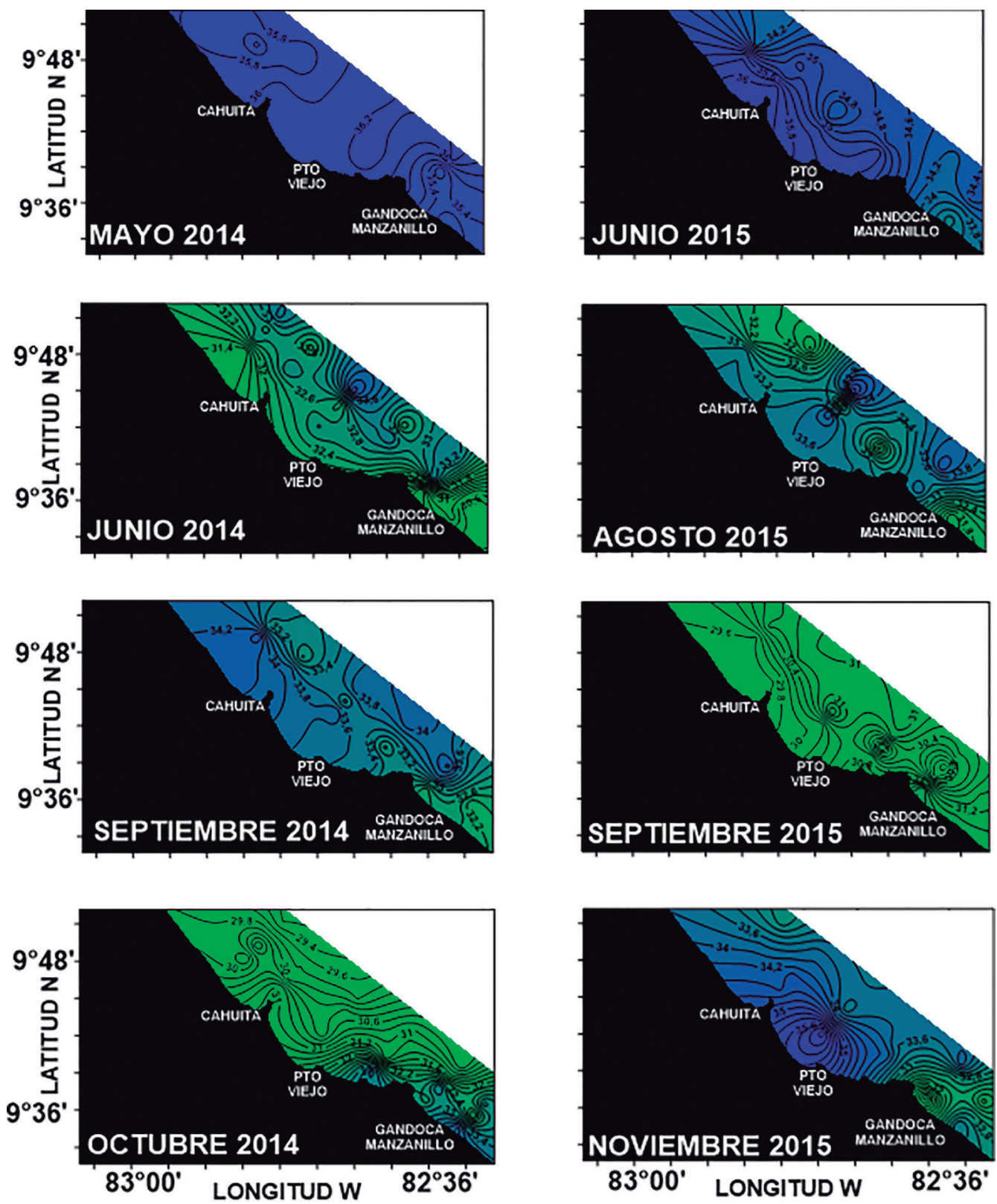

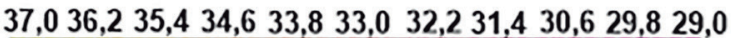

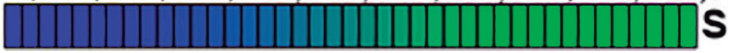

Fig. 4. Distribuciones superficiales de salinidad para los meses de muestreo Fig. 4. Surface salinity distributions for the sampling months 

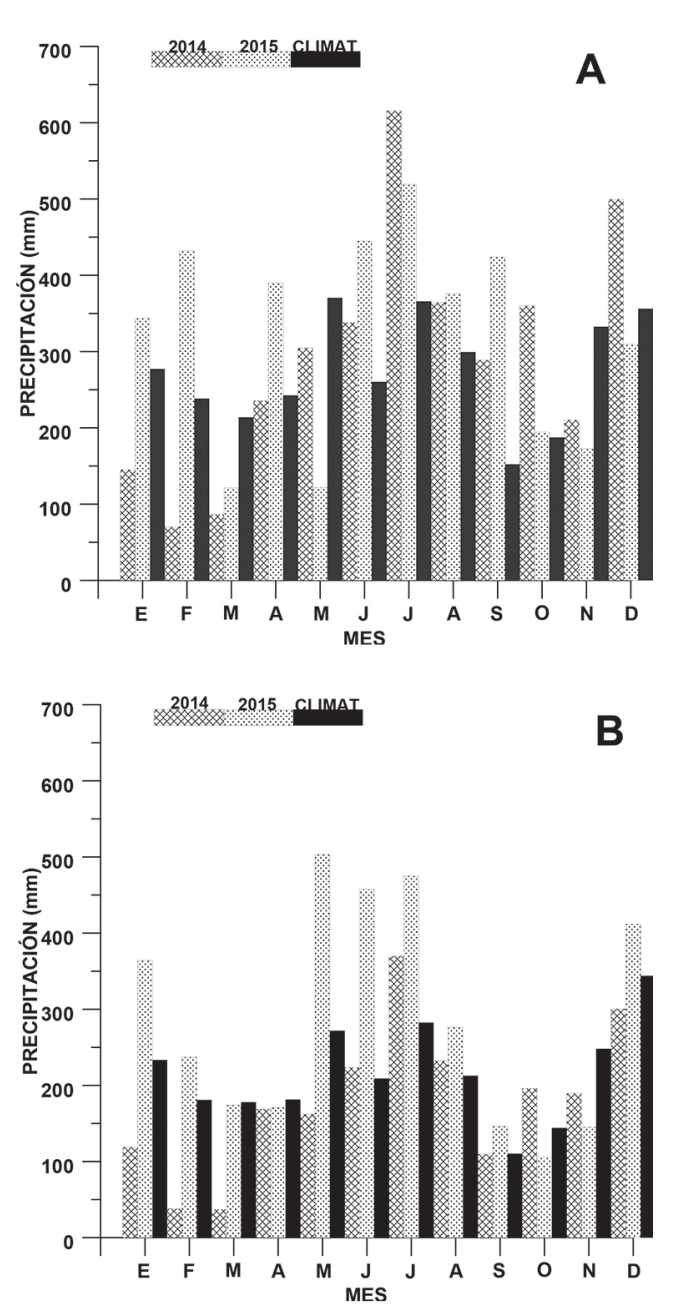

Fig. 5. Precipitación mensual ( $\mathrm{mm})$ en las estaciones de Puerto Vargas (A) y Sixaola (B) durante el 2014 y 2015 y climatología (mm)

Fig. 5. Monthly precipitation ( $\mathrm{mm})$ in Puerto Vargas (A) and Sixaola (B) stations during 2014 and 2015 and climatology $(\mathrm{mm})$

$\mathrm{mg} \mathrm{m}^{-3}$. En junio del $2014 \mathrm{y}$ agosto del 2015 los valores más altos se localizaron entre la superficie y los 10 $\mathrm{m}$ de profundidad. Fuera de la zona de ese máximo, la clorofila $a$ varió entre 0.1 y $0.3 \mathrm{mg} \mathrm{m}^{-3}$ en la superficie $\mathrm{y}$ el fondo, respectivamente.

La serie temporal (2002-2017) de la TSM del punto ubicado a 15 $\mathrm{km}$ frente a Cahuita permitió obtener su ciclo estacional con su máximo centrado en septiembre (Fig. 10A). La Figura 10B presenta las series de tiempo correspondientes al Índice Oceánico de El Niño (ONI, por sus siglas en inglés), el cual representa un promedio trimestral móvil de las anomalías de la TSM para la región Niño 3.4, y el residuo de la serie correspondiente a variaciones de la TSM con periodicidad entre 2 y 7 años. Se observa una concordancia entre ambas series, destacándose aquellos años en los cuales ocurrieron los Niños más recientes (2009-2010, 2014-2016). Se obtuvo una señal de calentamiento con un desfase de 7 meses posterior a la presencia de El Niño en el Océano Pacífico Tropical (Fig. 10C, Cuadro 1).

Adicionalmente, las anomalías de la TSM fueron obtenidas de la NOAA (2017) y se presentan en la Figura 11. A partir de septiembre del 2014 se observa una anomalía positiva superior a los $2^{\circ} \mathrm{C}$ en todo el Pacífico Centroamericano, sin embargo, la zona caribeña de Costa Rica mostraba un calentamiento muy débil, entre $0 \mathrm{y}$ $0.5^{\circ} \mathrm{C}$, y en algunos meses como junio del 2015 la anomalía fue negativa.

En el diagrama dispersivo T-S de todas las mediciones realizadas durante los muestreos, se pueden observar las 

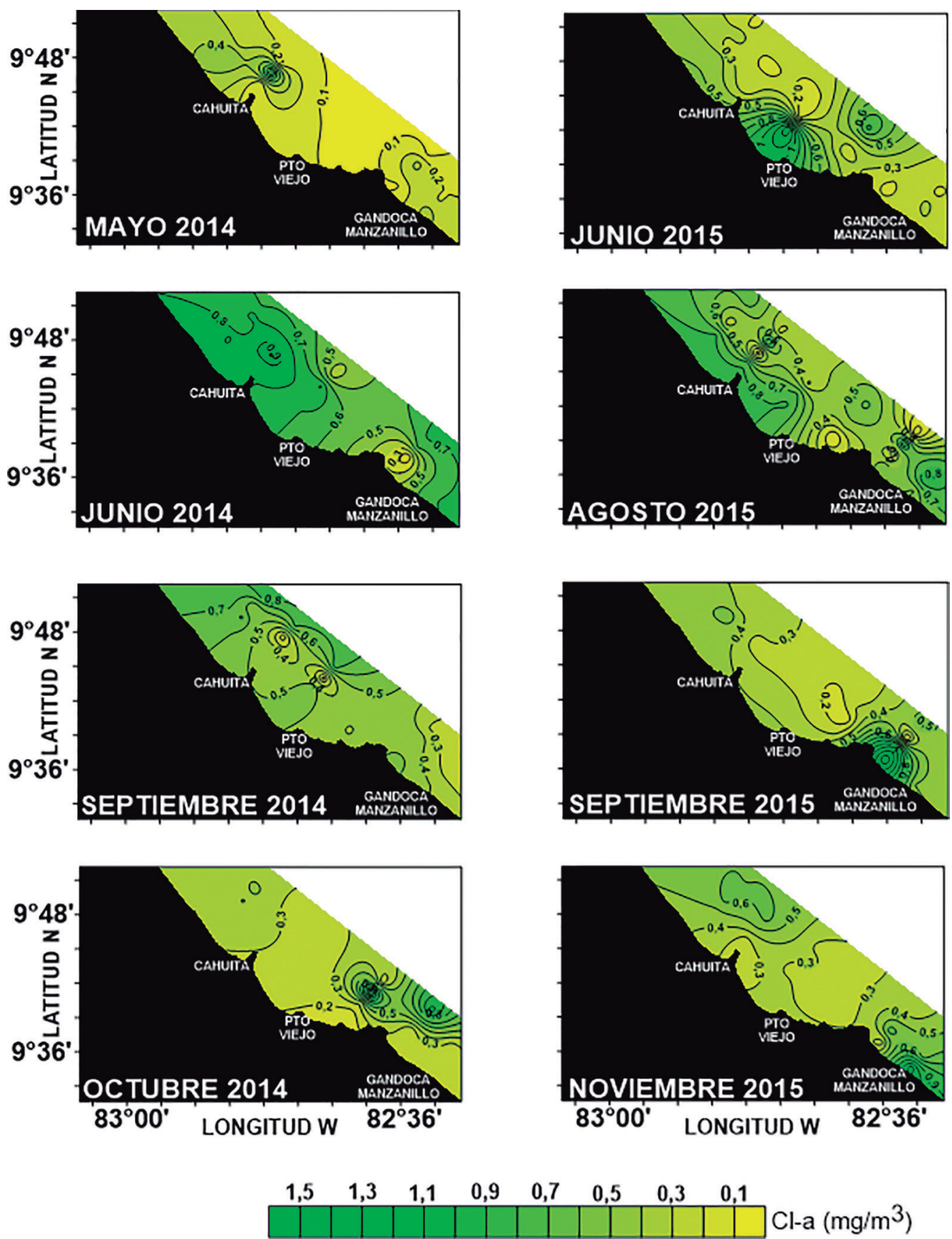

Fig. 6. Distribuciones superficiales de clorofila $a\left(\mathrm{mg} \mathrm{m}^{-3}\right)$ para los meses de muestreo Fig. 6. Surface distributions of chlorophyll $a\left(\mathrm{mg} \mathrm{m}^{-3}\right)$ for the sampling months 

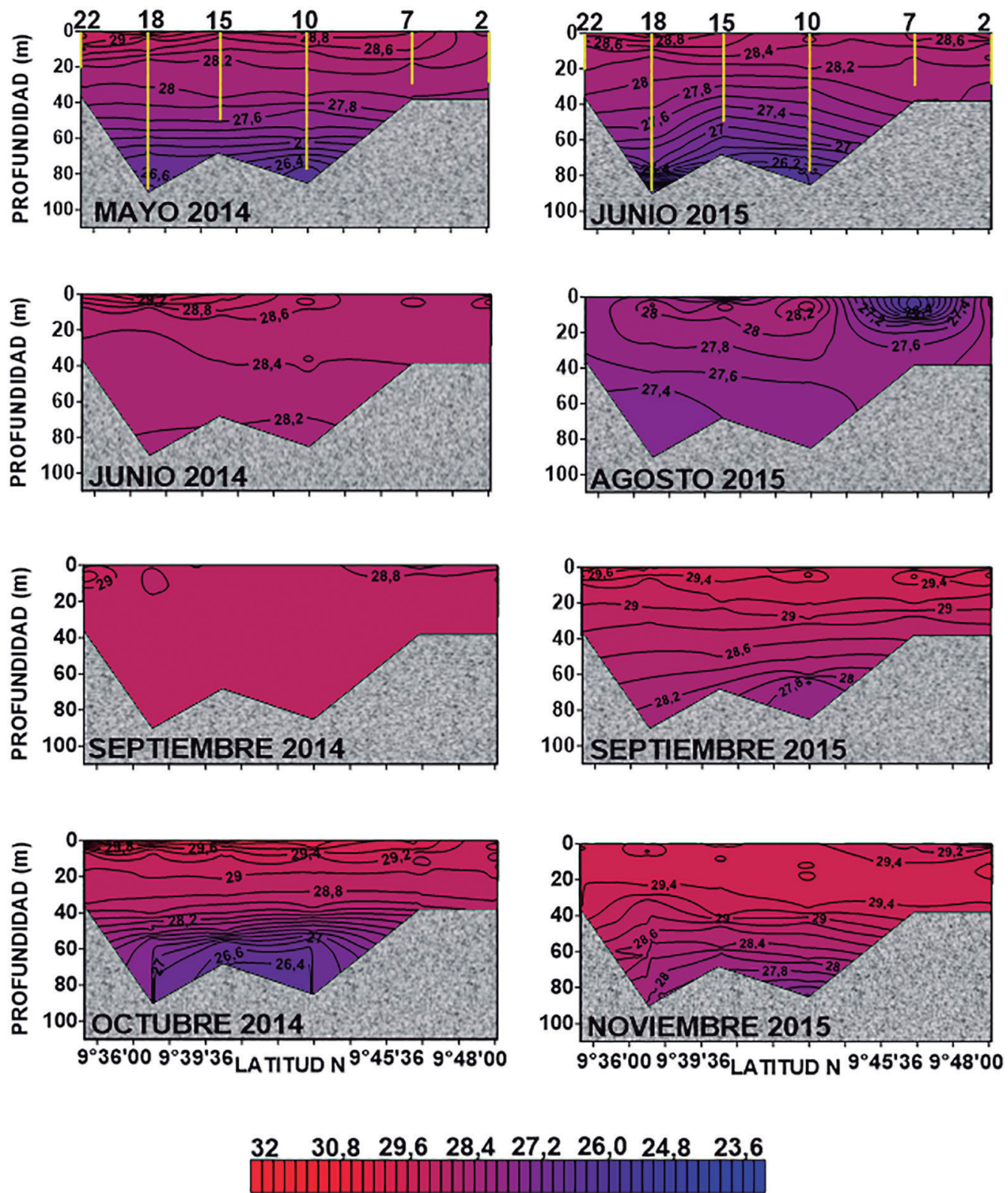

Fig. 7. Distribuciones verticales de la temperatura $\left({ }^{\circ} \mathrm{C}\right)$ en un transecto paralelo a la costa correspondientes a los meses de muestreo

Fig. 7. Vertical temperature distributions $\left({ }^{\circ} \mathrm{C}\right)$ in a transect parallel to the coast corresponding to the sampling months 

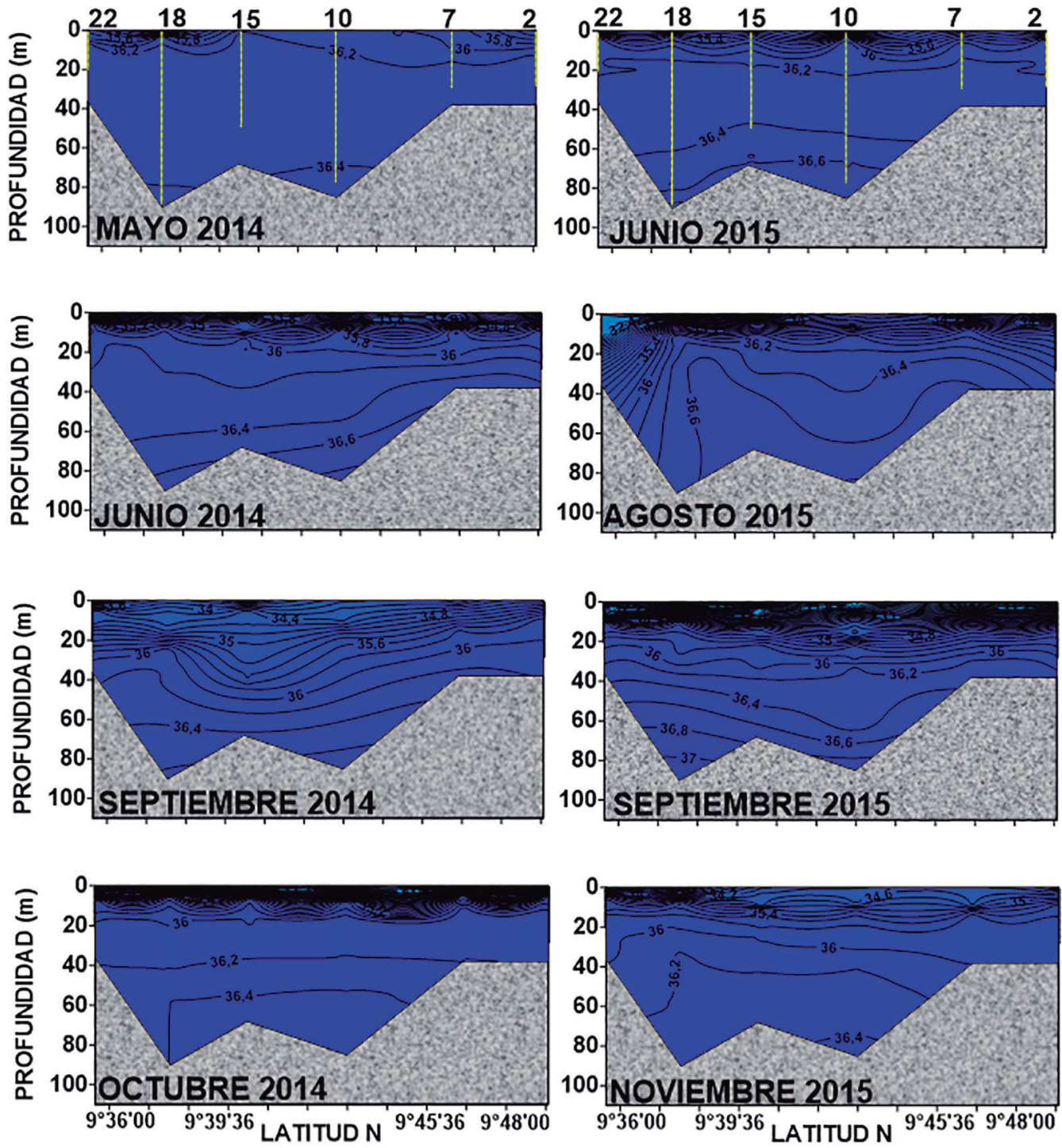

\section{$\begin{array}{llllll}36,8 & 35,2 & 33,6 & 32,0 & 30,4 & 28,8\end{array}$}

Fig. 8. Distribuciones verticales de la salinidad en un transecto paralelo a la costa correspondientes a los meses de muestreo

Fig. 8. Vertical distributions of salinity in a transect parallel to the coast corresponding to the sampling months 
variaciones experimentadas por los índices termohalinos de la masa de agua superficial durante todo el período de estudio (Fig. 12). Por debajo de los $28^{\circ} \mathrm{C}$ la dispersión de los pares T-S es muy pequeña.

\section{DISCUSIÓN}

El ONI mostró sus valores más elevados al final del 2015 y principios del 2016, de modo que la fase madura de este episodio cálido se localizó entre septiembre y marzo de esos años, debilitándose a partir de abril del 2016. Si bien es cierto las aguas superficiales en el área de interés presentaron las temperaturas más elevadas en septiembre y noviembre del 2015 (Figs. 2 y 3), ese calentamiento forma parte del ciclo anual de dicha variable (Fig. 10A). Este comportamiento estacional coincide con lo reportado por otros autores para la Cuenca Colombiana del Mar Caribe (Amador et al. 2006; RuizOchoa, 2011; Ruiz-Ochoa et al. 2012; Amador et al.2016), en el cual de mayo a septiembre las aguas muestran su máximo calentamiento. A partir de este último mes y hasta los primeros meses del año el campo térmico superficial experimenta un enfriamiento asociado principalmente a la variación en los campos de viento por la influencia de frentes fríos al final y principio de cada año y menor radiación incidente (Amador et al. 2006; 2016). Existe, por lo tanto, estacionalmente un calentamiento progresivo a partir de junio alcanzando su máximo entre septiembre y noviembre. La región estudiada no exhibió en términos superficiales un gradiente espacial térmico significativo.

Las altas temperaturas superficiales observadas en los meses de septiembre y noviembre no corresponden directamente a la presencia de El Niño, toda vez que las anomalías de la TSM fueron muy cercanas a $0^{\circ} \mathrm{C}$, e inclusive, negativas en junio y agosto del 2015 (Fig. 11). En un trabajo realizado por Bernal et al. (2006) se analizó la influencia de El Niño sobre la TSM en el Caribe Colombiano incluido el extremo sur de Panamá (Golfo de Darién). Los autores determinaron a partir de una serie temporal de 20 años (1981-2000), que las correlaciones cruzadas entre el MEI (siglas en inglés del Índice Multivariado del ENSO) y el SOI (siglas en inglés del Índice de la Oscilación del Sur) presentaban un rezago de 7 y 6 meses, respectivamente, con respecto a las series de la TSM estandarizadas. Un resultado similar fue obtenido también por Enfield \& Mayer (1997).

En el presente estudio se obtuvo un resultado semejante al correlacionar las variaciones de la TSM con periodicidades entre 2 y 7 años y el ONI. Como se comentó previamente, la zona estudiada presentó una señal de calentamiento con un desfase de 7 meses posterior a la presencia de El Niño (Fig. 10C, Cuadro 1). Este 

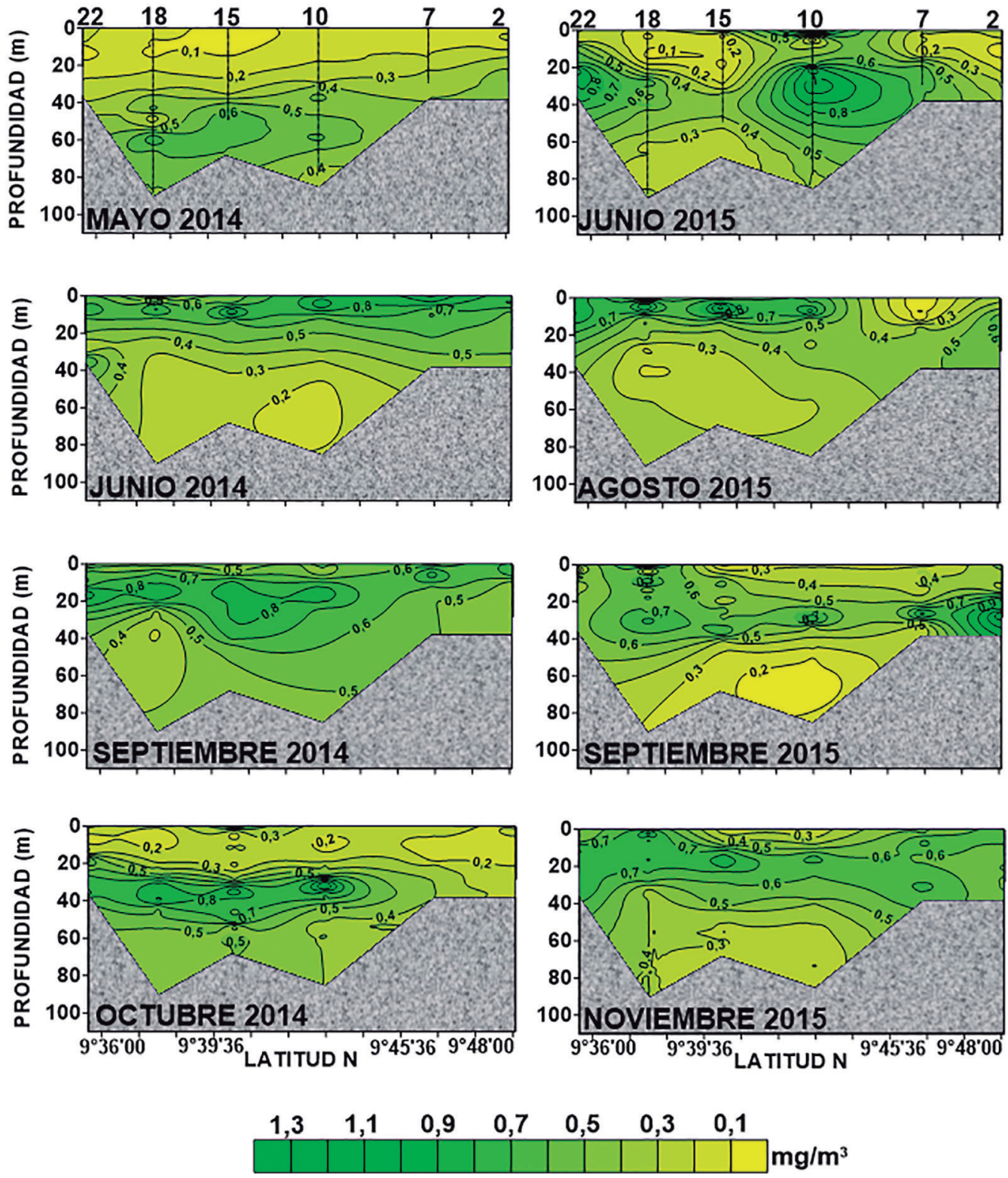

Fig. 9. Distribuciones verticales de clorofila $a\left(\mathrm{mg} \mathrm{m}^{-3}\right)$ en un transecto paralelo a la costa correspondientes a los meses de muestreo

Fig. 9. Vertical distributions of chlorophyll $a\left(\mathrm{mg} \mathrm{m}^{-3}\right)$ in a transect parallel to the coast corresponding to the sampling months 

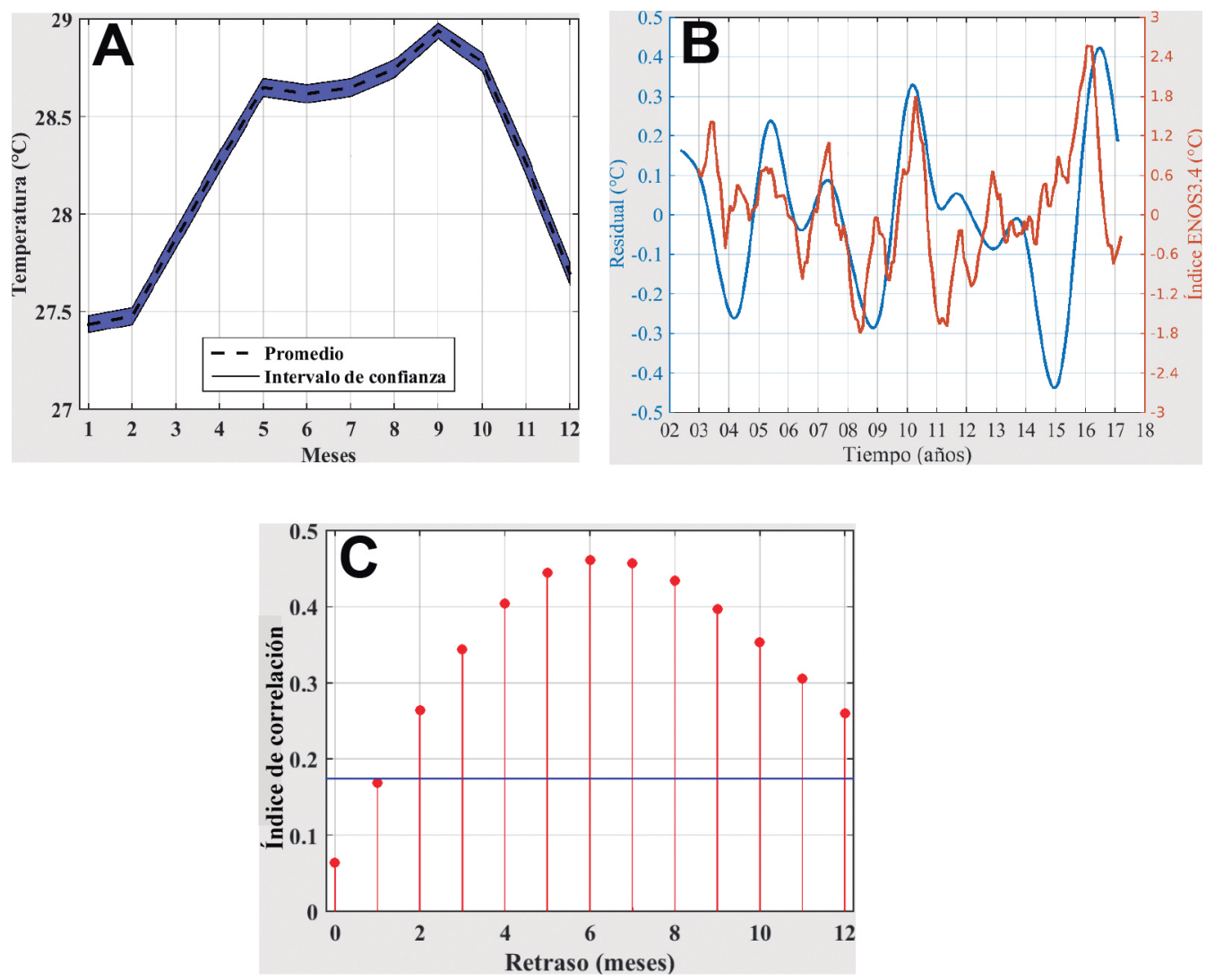

Fig. 10. Ciclo anual de la temperatura superficial del mar $\left({ }^{\circ} \mathrm{C}\right)(\mathrm{A})$, series temporales del Î́ndice Oceánico de El Niño (ONI) y de la señal de la temperatura superficial del mar con periodicidad entre 2 y 7 años $\left({ }^{\circ} \mathrm{C}\right)(\mathrm{B})$, índice de correlación entre las dos series anteriores, para un punto oceánico ubicado a $15 \mathrm{~km}$ de Cahuita, Limón, Costa Rica $(\mathrm{C})$ Fig. 10. Annual cycle of surface temperature $\left({ }^{\circ} \mathrm{C}\right)(\mathrm{A})$, time series of the Oceanic El Niño Index (ONI) and the sea surface temperature signal with a periodicity between 2 and 7 years $\left({ }^{\circ} \mathrm{C}\right)(\mathrm{B})$, and correlation index between the two previous series, for an oceanic point located $15 \mathrm{~km}$ from Cahuita, Limón, Costa Rica (C)

calentamiento se caracteriza por una anomalía en la TSM entre $0.4^{\circ} \mathrm{C}$ y $0.5^{\circ} \mathrm{C}$ (Fig. 10B).

Spillman et al. (2011) encontraron que el calentamiento que experimenta el Mar Caribe 7 meses después de la ocurrencia de un Niño, coincide con reportes previos de blanqueamiento de corales en esta zona, poniendo de relieve su influencia motriz en los meses posteriores a un evento, lo cual se refleja en la ocurrencia de la mayoría de los episodios de blanqueamiento en masa durante períodos fuertes de El Niño. McWilliams et al. (2005) estiman que un calentamiento regional del Mar Caribe del orden de $0.1^{\circ} \mathrm{C}$ representaría un aumento entre el $35 \%$ y $42 \%$ en la extensión geográfica e intensidad en el blanqueamiento de corales. En junio 
Cuadro 1. Índice de correlación y significancia para las series temporales del Índice Oceánico de El Niño (ONI) y la señal de la temperatura superficial del mar con una periodicidad entre 2 y 7 años

Table 1. Correlation index and significance for the time series of the Oceanic El Niño Index (ONI) and the signal of surface temperature with a periodicity between 2 and 7 years

\begin{tabular}{ccc}
\hline Retraso (meses) & $\begin{array}{c}\text { Correlación } \\
(\mathbf{r})\end{array}$ & $\begin{array}{c}\text { Significancia } \\
(\boldsymbol{P})\end{array}$ \\
\hline 0 & 0.0465 & 0.5396 \\
1 & 0.1456 & 0.0586 \\
2 & 0.2364 & 0.0019 \\
3 & 0.3156 & $2.67 \mathrm{E}-05$ \\
4 & 0.3807 & $2.77 \mathrm{E}-07$ \\
5 & 0.4279 & $3.86 \mathrm{E}-09$ \\
6 & 0.4595 & $1.76 \mathrm{E}-10$ \\
7 & 0.4742 & $3.13 \mathrm{E}-11$ \\
8 & 0.4730 & $2.99 \mathrm{E}-11$ \\
9 & 0.4575 & $1.30 \mathrm{E}-10$ \\
10 & 0.4288 & $1.68 \mathrm{E}-09$ \\
\hline
\end{tabular}

del 2015 ya se alertaba sobre el aumento en la amenaza de blanqueo de corales en el Caribe debido a El Niño 2014-2016 (Kennedy, 2015).

La variación temporal observada en el calentamiento de las aguas costeras entre Cahuita y Manzanillo puede explicarse posiblemente en función del aumento en las descargas de agua dulce procedentes de las escorrentías, las cuales se incrementan a partir del segundo semestre en concordancia con el ciclo anual de precipitaciones, y aún más en presencia de El Niño. Estas descargas producen salinidades superficiales bajas (Fig. 4) de modo que las aguas costeras serán menos densas en este período. La fuerte picnoclina (no mostrada aquí) asociada a la estratificación vertical del campo salino se comporta como una barrera difusiva que inhibe la mezcla vertical, permitiendo de esta manera la absorción de la radiación solar en la fina capa superficial. Esto explicaría la concordancia registrada entre los valores de la TSM obtenidos in situ y los derivados de las observaciones satelitales (Figs. 2 y 3 ).

Las salinidades en la capa superficial (Fig. 4) son en la mayoría de los meses inferiores a las que se han observado en esa región $(34.2<\mathrm{S}<$ 35.4) durante períodos en los cuales El Niño ha estado ausente (BrenesRodríguez \& Benavides-Morera, 2015). Esta es quizás la principal huella de este fenómeno durante su 

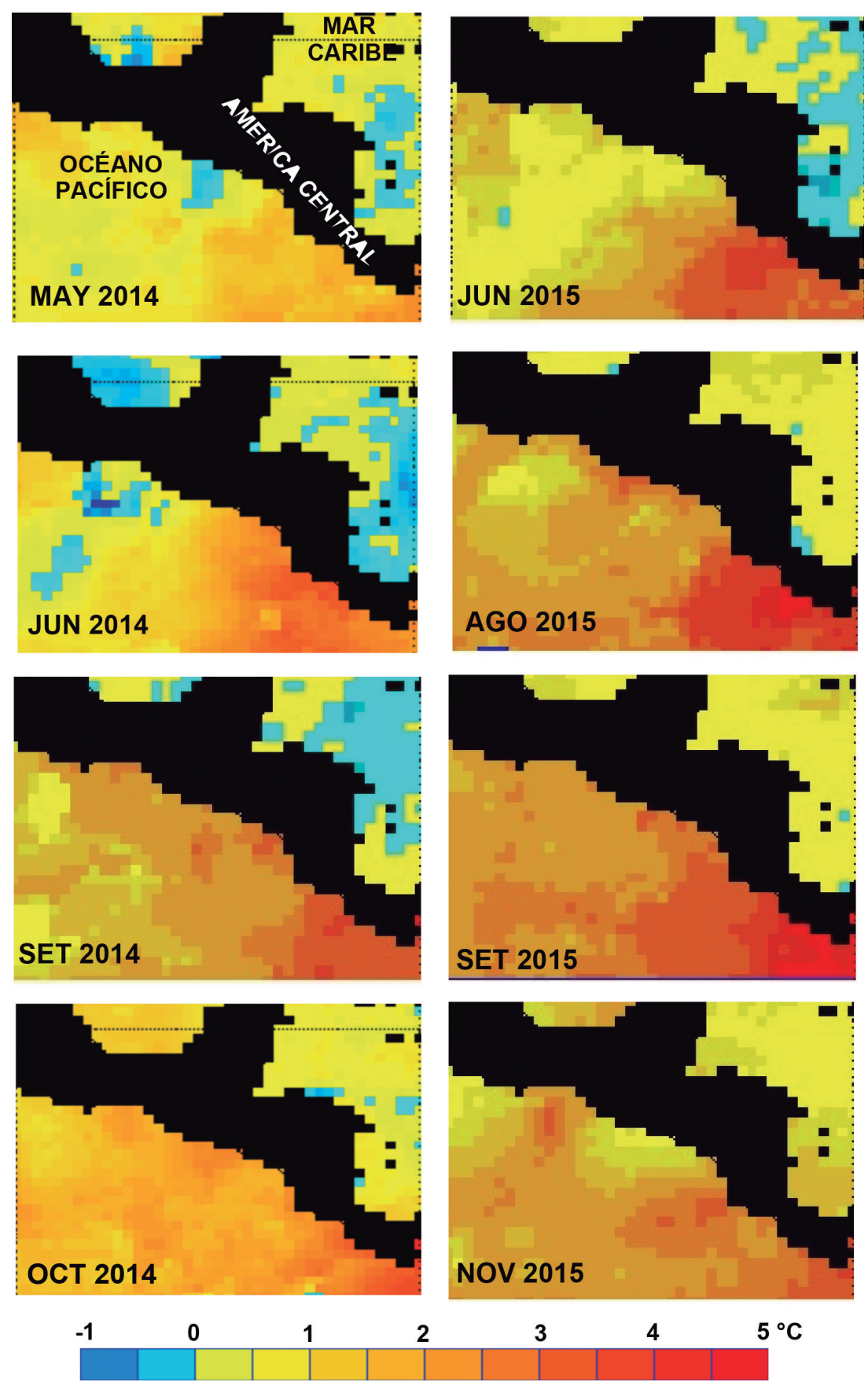

3

Fig. 11. Anomalías de la TSM $\left({ }^{\circ} \mathrm{C}\right)$ para los meses de estudio (NOAA, 2017) Fig. 11. SST anomalies $\left({ }^{\circ} \mathrm{C}\right)$ for the study months (NOAA, 2017) 


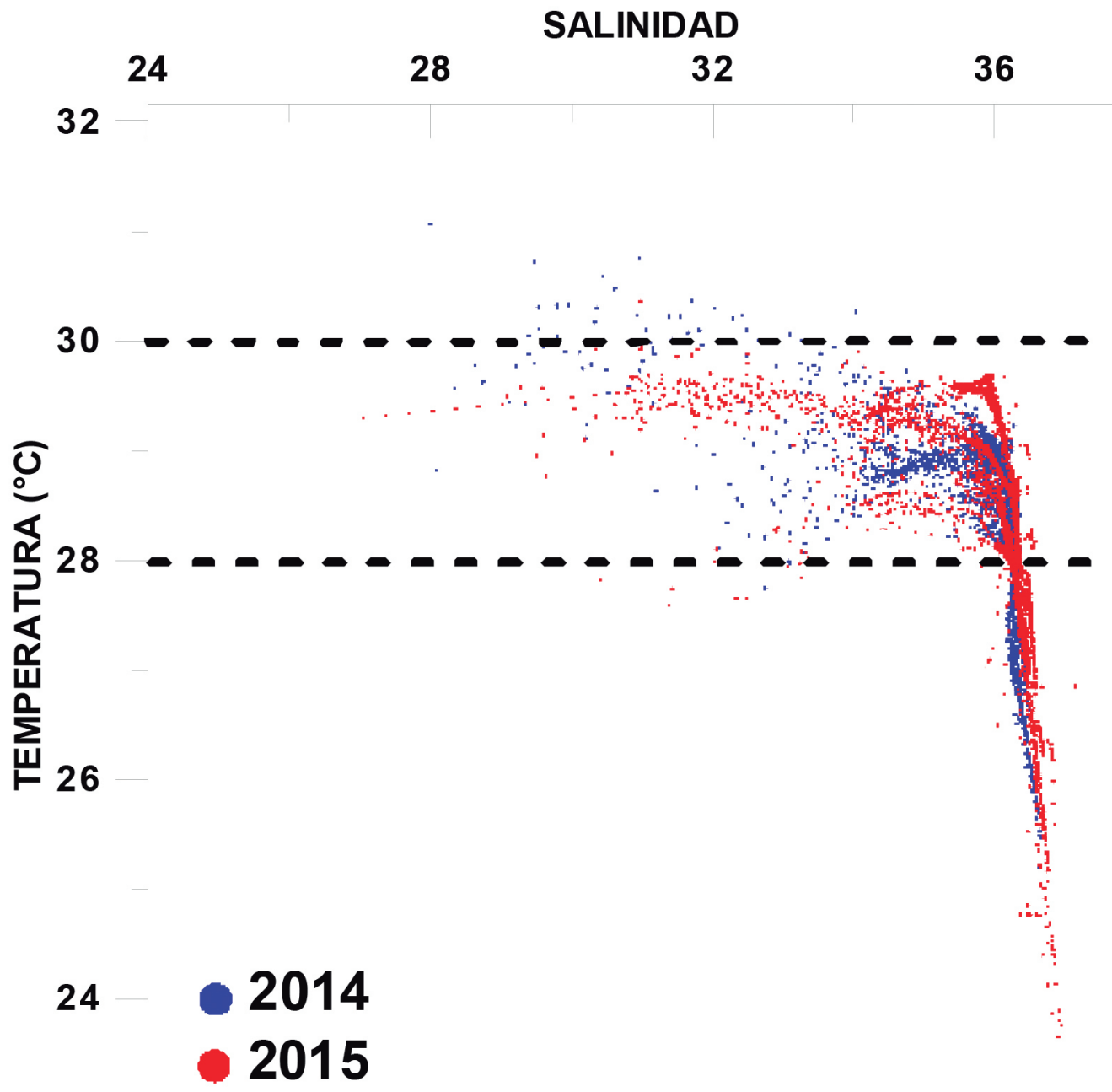

Fig. 12. Diagrama dispersivo T-S para todas las estaciones durante el período de muestreo. Las líneas discontinuas muestran los límites térmicos del Agua Superficial del Caribe

Fig. 12. Dispersive T-S diagram for all stations during the sampling period. The broken lines show the thermal limits of the Surface Water of the Caribbean

desarrollo sobre la hidrografía del área. Las precipitaciones en el Caribe de Costa Rica se caracterizan por presentar dos períodos lluviosos muy definidos (Alfaro, 2002). El primero de ellos ocurre entre junio y agosto $y$ está asociado a la intensificación de los alisios y la corriente en chorro de bajo nivel del Caribe (CLLJ, por sus siglas en inglés, Amador, 2008), mientras que la presencia de frentes fríos son los responsables del segundo período 
1luvioso de noviembre a enero (ZárateHernández, 2013).

La precipitación en el Caribe de Costa Rica presenta un acentuado gradiente latitudinal (norte-sur), con marcadas diferencias entre el Caribe Norte (Tortuguero) y el Caribe Sur (Sixaola) (IMN, 2008; Pérez-Briceño et al. 2017). Este comportamiento se observa también en el área estudiada, en la cual las estaciones meteorológicas de Puerto Vargas (Cahuita) y Sixaola separadas una distancia cercana a los 10 $\mathrm{km}$, presentan en algunos casos para un mismo mes diferencias en los registros de lluvia entre el 10\% y $40 \%$ (Fig. 5 A y B). Entre junio del 2014 y septiembre del 2015 las precipitaciones estuvieron por encima de lo normal, con excepción de algunos meses. En el promedio anual la diferencia es de unos $550 \mathrm{~mm}$ de lluvia más en Puerto Vargas. Este comportamiento tan particular asociado a la presencia de El Niño tuvo una influencia directa en el comportamiento de los campos salinos superficiales.

Los frentes fríos responsables principales del aumento de precipitación que se observa entre diciembre y febrero (Fig. 5 A y B) se distribuyen principalmente entre los últimos y los primeros tres meses de cada año. El caso del pico lluvioso observado entre mayo y julio obedece a una intensificación del viento alisio más fuerte de lo normal, característico de años de El Niño (Vega \& Stolz, 1997). Para la vertiente del Caribe, el 93\% de los eventos extremos lluviosos están asociados a la presencia de El Niño (IMN, 2008).
La influencia de las descargas de los ríos Estrella al norte de Cahuita y el Sixaola al sur de Gandoca se ve reflejada en las distribuciones superficiales de clorofila $a$ (Fig. 6). Las mayores concentraciones $(>0.4$ $\mathrm{mg} \mathrm{m}^{-3}$ ) se observan cerca de las áreas antes citadas, en zonas que coinciden con la presencia de aguas de baja salinidad (Fig. 4). En general, la zona mostró condiciones mesotróficas $(0.2$ $\mathrm{mg} \mathrm{m}^{-3}<\mathrm{Chl} a<1 \mathrm{mg} \mathrm{m}^{-3}$ ).

Brenes-Rodríguez \& BenavidesMorera (2015) habían evidenciado una situación similar en un trabajo previo realizado en todo el Caribe Sur. El transporte de nutrientes inorgánicos a través de descargas fluviales ha sido reportado como el responsable del aumento de nutrientes en zonas costeras cercanas a las desembocaduras de ríos (Marchetti et al. 1989; Justic et al. 1995; Rabalais et al. 1996; Olivos et al. 2002; Restrepo et al. 2006). Los valores superficiales más bajos de clorofila $a$ se obtuvieron en mayo del 2014, un mes con una precipitación relativamente baja. En ese mismo mes el máximo subsuperficial se localizó en su posición más profunda $(\mathrm{Z} \geq 60 \mathrm{~m})$ y las concentraciones de clorofila $a$ en los primeros $20 \mathrm{~m}$ de la columna de agua fueron muy bajas $\left(\sim 0.1 \mathrm{mg} \mathrm{m}^{-3}\right)$. Todo parece indicar que el ensanchamiento y la posición vertical de dichos máximos desde la superficie hasta los $40 \mathrm{~m}$, tienen una relación directa con el aumento en las precipitaciones asociadas en nuestro caso a la presencia de El Niño de esa época. De esta forma, el patrón local 
de escorrentías (en concordancia con el ciclo de precipitaciones) podría ser el principal mecanismo de fertilización de la región estudiada, influyendo en la disponibilidad de nutrientes $\mathrm{y}$, por ende, en la biomasa fitoplanctónica expresada en forma de clorofila $a$.

Por último, el diagrama dispersivo T-S (Fig. 12) evidencia que el ASC (Agua Superficial del Caribe), cuyos índices termohalinos se caracterizan por salinidades relativamente bajas (34.5-36) y altas temperaturas (28$30^{\circ} \mathrm{C}$ ) (Mooers \& Maul, 1998; Andrade \& Barton, 2000; Hernández-Guerra \& Joyce, 2000), experimentó con la influencia de El Niño una dilución considerable con salinidades inferiores a 32, mientras que su temperatura aumentó en forma leve por encima de $\operatorname{los} 30^{\circ} \mathrm{C}$. Bajo la superficie para aguas con temperaturas inferiores a $28.5^{\circ} \mathrm{C}$, las curvas T-S son muy similares entre los meses muestreados en el 2014 y el 2015, y no se observa ningún calentamiento interanual asociado a El Niño. La mayor dispersión en los puntos del diagrama T-S se localiza en la superficie, donde los factores hidrometeorológicos tienen su mayor influencia.

\section{AGRADECIMIENTOS}

Este trabajo se realizó gracias al apoyo de la Universidad Nacional de Costa Rica a través del Proyecto "Servicio Regional de Información Oceanográfica" del Departamento de Física, y del Proyecto "Indicadores ambientales en ecosistemas marinos y costeros para la definición de estrategias de conservación y manejo en dos áreas protegidas en el Caribe Sur de Costa Rica", de la Escuela de Ciencias Biológicas. Nuestros agradecimientos a Lilliana Piedra, coordinadora del último proyecto mencionado, y a Jorge Cortés del Centro de Investigación en Ciencias del Mar y Limnología (CIMAR), Universidad de Costa Rica, por sus valiosos comentarios. Los autores agradecemos a los revisores anónimos por la revisión detallada al manuscrito, lo cual contribuyó a enriquecer la información presentada.

\section{BIBLIOGRAFÍA}

Alfaro, E. (2002). Some Characteristics of the Annual Precipitation Cycle in Central America and their Relationship with its Surrounding Tropical Oceans. Top. Meteor. Oceanogr., 9(2), 88-103. Amador, J. A., Alfaro, E. J., Lizano, O. G. \& Magaña, V. O. (2006). Atmospheric forcing of the Eastern tropical Pacific: A review. Progr. Oceanogr., 69(2-4), 101-142. doi: https://doi.org/10.1016/j. pocean.2006.03.007

Amador, J. A. (2008). The Intra-Americas Sea Low-level Jet Overview and Future Research. Ann. N. Y. Acad. Sci., 1146(1), 153-188. doi: http://doi. org/10.1196/annals. 1446.012

Amador, J. A., Durán-Quesada, A. M., Rivera, E. R., Mora, G., Sáenz, F., Calderón, B. \& Mora, N. (2016). The easternmost tropical Pacific. Part II: Seasonal and interseasonal modes of atmospheric variability. Rev. Biol. Trop., 64(Suppl. 1), S23-57. doi: https://doi. org/10.15517/rbt.v64i1.23409 
Andrade, C. \& Barton, E. (2000). Eddy development and motion in the $\mathrm{Ca}$ ribbean Sea. J. Geophys. Res., 115(C11), 26191-26201. doi: http:// dx.doi.org/10.1029/2000JC000300

Bernal, G., Poveda, G., Roldán, P. \& Andrade, C. (2006). Patrones de variabilidad de las temperaturas superficiales del mar en la costa Caribe colombiana. Rev. Acad. Colomb. Cien., XXX(115), 195-208.

Brenes-Rodríguez, C. L. \& BenavidesMorera, R. (2015). Características termohalinas de las aguas costeras del Caribe Sur de Costa Rica. Rev. Mar. Cost., 7, 27-41. doi: http://dx.doi. org/10.15359/revmar.7.2

Chin, T. M., Vázquez, J. \& Armstrong, E. (2013). Algorithm Theoretical Basis Document: A multi-scale, high-resolution analysis of global sea surface temperature, Version 1, 13. Recuperado en julio 26, 2017, disponible enftp:// mariana.jpl.nasa.gov/mur_sst/tmchin/ docs/ATBD/atbd_1.1.pdf

Cortés, J., Murillo, M. M., Guzmán, H. M. \& Acuña, J. (1984). Pérdida de zooxantelas y muerte de corales y otros organismos arrecifales en el Caribe y Pacífico de Costa Rica. Rev. Biol. Trop., 32, 227-232.

Cortés, J. (2016). The Caribbean coastal and marine ecosystem. In M. Kapelle (Ed.), Costa Rican Ecosystems (pp. 591-617). Chicago, IL, EE.UU.: University of Chicago Press.

Enfield, D. \& Mayer. D. A. (1997). Tropical Atlantic sea surface temperature variability and its relation to El-Niño-Southern Oscillation. J. Geophys. Res., 102, 929-945. doi: https://doi. org/10.1029/96JC03296

Hernández-Guerra, A. \& Joyce, T. M. (2000). Water masses and circulation in the surface layers of the
Caribbean at $66^{\circ} \mathrm{W}$. Geophys. Res. Lett., 27(21), 3497-3500. doi: https:// doi.org/10.1029/1999GL011230

IMN. (2008). Instituto Meteorológico Nacional. Clima, Variabilidad y Cambio Climático en Costa Rica. Proyecto Segunda Comunicación Nacional de Costa Rica. PNUD-CRRH-MINAE, San José, Costa Rica. Recuperado en junio 4, 2017, disponible en http://cglobal.imn.ac.cr/documentos/ clima-variabilidad-y-cambio-climatico IMN. (2016). Instituto Meteorológico Nacional. Pronóstico Nacional. Pronóstico Fenómeno ENOS y estación lluviosa 2016. Folleto informativo. San José, Costa Rica. Recuperado en junio 14, 2017, disponible en https://www. google.com/search?client=firefox-b$\mathrm{ab} \& \mathrm{q}=$ pron $\% \mathrm{C} 3 \% \mathrm{~B} 3$ stico + fen $\% \mathrm{C} 3$ $\% \mathrm{~B} 3$ meno+enos $+\mathrm{y}+\mathrm{estaci} \% \mathrm{C} 3 \% \mathrm{~B} 3$ $\mathrm{n}+$ lluviosa $+2016 \&$ oq $=\& \mathrm{gs} \_\mathrm{l}=\mathrm{psy}-\mathrm{ab}$ $.1 .2 .35 \mathrm{i} 39 \mathrm{k} 116.2556 .2556 .0 .6636 .1 .1$ $.0 .0 .0 .0 .155 .155 .0 \mathrm{j} 1.1 .0 \ldots . .0 \ldots 1.1 .64$. psy-ab..0.1.154.6.3Eg2c3U_HHA

Jiménez, C. (2001). Bleaching and mortality of reef organisms during a warming event in 1995 on the Caribbean coast of Costa Rica. Rev. Biol. Trop., 49(Suppl. 2), 233-238.

Justic, D., Rabalais, N. N., Turner, R. E. \& Dortch, Q. (1995). Changes in nutrient structure of river-dominated coastal waters: Stoichiometric nutrient balance and its consequences. Estuar, Coast. Shelf Sci., 40(3), 339-356. doi: https://doi. org/10.1016/S0272-7714 (05)80014-9

Kennedy, K. (2015). El Niño revs up coral bleaching threat in the Caribbean. Recuperado en mayo 20, 2017, disponible en https://www.climate.gov/ news-features/featured-images/elni\%C3\%B1o-revs-coral-bleachingthreat-caribbean. 
Marchetti, R., Provini, A. \& Crosa, G. (1989). Nutrient load carried by the River Po into the Adriatic Sea, 1968-1987. Mar. Poll. Bull., 20, 168-172. doi: https://doi. org/10.1016/0025-326X(89)90487-6

McWilliams, J. P., Coté, I. M., Gill, J. A., Sutherland, W. J. \& Watkinson, A. R. (2005). Accelerating impacts of temperature-induced coral bleanching in the Caribbean. Ecology, 86(8), 2055-2060. doi: https://doi.org/10.1890/04-1657

Mooers, C. \& Maul, G. (1998). Intra-Americas Sea circulation. In H. Brink. \& A. Robinson (Eds.), The Sea: The Global Coastal Ocean (pp. 183-208). New Jersey, EE.UU.: Wiley \& Sons, Inc.

NOAA. (2016). National Oceanographic and atmospheric Agency. June 2016 ENSO discussion: The new neutral. Recuperado en noviembre 21, 2017, disponible en https:/www.climate.gov/news-features/ blogs/enso/june-enso-discussion-newneutral\#.V1mjj0DiZ-A.facebook

NOAA. (2017). National Oceanographic and atmospheric Agency. GOES Project Science. Recuperado en junio 5, 2017, disponible en http://rsd.gsfc. nasa.gov/goes/

Olivos, A., Quijano, S. I. García, G., Galicia, M. A. \&Gaviño, J. H. (2002). Influencia de los parámetros fisicoquímicos en la distribución de los principales grupos fitoplanctónicos en las costas del Estado de Colima durante el 2002. Ciencia en la Frontera: Revista de Ciencia y Tecnología de la UACJ, 3(1), 13-28.

Pérez-Briceño, P. M., Amador-Astúa, E. J. \& Alfaro, E. (2017). Dos propuestas de clasificación climática para la vertiente Caribe costarricense según el sistema de Thornthwaite. Rev. Clim., 17, 1-16.

Rabalais, N. N., Turner, R. E., Justić, D., Dortch, Q., Wiseman, W. J. \& Gupta, B. K. S. (1996). Nutrient changes in the Mississippi River and system responses on the adjacent shelf. Estuaries, 19(2), 386-407. doi: https://doi. org/10.2307/1352458

Restrepo, J., Zapata, P., Díaz, J., GarzónFerreira, J. \& García, C. (2006). Fluvial fluxes into the Caribbean Sea and their impact on coastal ecosystems: The Magdalena River, Colombia. Global Planet Change, 50, 33-49. doi: http:// doi:10.1016/j.gloplacha.2005.09.002

Ruiz-Ochoa, M. A. (2011). Variabilidad de la Cuenca Colombia (mar Caribe) asociada con El Niño-Oscilación del Sur, vientos Alisios y procesos locales. Tesis de doctorado no publicada, Universidad Nacional de Colombia.

Ruiz-Ochoa, M., Beier, E., Bernal, G. \& Desmond, E. (2012). Sea surface temperature variability in the Colombian Basin, Caribbean Sea. Deep-Sea Res., I(64), 43-53. doi: https://doi. org/10.1016/j.dsr.2012.01.013

Spillman, C. M., Alves, O. \& Hudson, A. (2011). Seasonal prediction of thermal stress accumulation for coral bleachingin the tropical oceans. Month. Weather Rev., 39, 318-331.

SURFER 14. (2017). Surfer ® Contouring and 3D Surface Mapping for Scientists and Engineers, version 14 www.GoldenSoftware.com. Colorado, EE.UU.: Golden Software, Inc.

Vega, G. \& Stolz, W. (1997). El fenómeno de "El Niño" y su impacto en la economía de Costa Rica. Ministerio del ambiente y Energía. San José, Costa Rica: Oficina de Pronósticos. Instituto Meteorológico Nacional.

Zárate-Hernández, E. (2013). Climatología de masas invernales de aire frío que alcanzan Centroamérica y el Caribe y su relación con algunos índices árticos. Top. Meteor. Oceanogr., 12(1), 35-55. 